\title{
Fréquentation et exploitation des sources salées (Var et Vaucluse)
}

\section{Audrey Boutet}

\section{OpenEdition Journals}

Édition électronique

URL : http://journals.openedition.org/adlfi/6613

ISSN : 2114-0502

Éditeur

Ministère de la culture

Référence électronique

Audrey Boutet, "Fréquentation et exploitation des sources salées (Var et Vaucluse) », ADLFI.

Archéologie de la France - Informations [En ligne], Provence-Alpes-Côte d'Azur, mis en ligne le 01 mars

2007, consulté le 19 avril 2019. URL : http://journals.openedition.org/adlfi/6613

Ce document a été généré automatiquement le 19 avril 2019

(C) Ministère de la Culture et de la Communication, CNRS 


\title{
Fréquentation et exploitation des sources salées (Var et Vaucluse)
}

\author{
Audrey Boutet
}

\author{
Identifiant de l'opération archéologique : 8216 \\ Date de l'opération : 2007 (PT) \\ Inventeur(s) : Boutet Audrey (AUT)
}

1 Cette opération de prospection thématique s'inscrit dans le cadre de nos travaux de recherche concernant l'exploitation du sel en Gaule méridionale de la fin de l'âge du Bronze à l'Antiquité tardive, effectués depuis deux ans dans le cadre d'un master et poursuivis depuis cette année dans le cadre d'un doctorat à l'université d'Aix-enProvence (centre Camille-Jullian).

2 Le sel est un produit indispensable à l'homme et a été exploité dès le Néolithique. Les sites d'exploitation du sel, marin ou de sources salées, sont bien connus en Gaule septentrionale mais très peu dans les régions méridionales (Boutet, 2006). Pourtant, le midi de la Gaule possède de riches ressources salifères et notamment de nombreux gisements de sel dans l'arrière-pays (sources salées principalement). La découverte récente d'aménagements néolithiques dans le puits salé de Moriez (Alpes-de-HauteProvence) a sur ce point démontré le potentiel régional pour une archéologie du sel continental (BSR PACA, 1999 : 30-32).

3 Le choix de notre étude s'est porté sur deux départements, le Var et le Vaucluse, qui possèdent un fort potentiel.

\section{Département du Var}

Des travaux documentaires avaient permis de montrer la richesse des ressources en sel continental du département, qu'il s'agisse de sources salées (communes de Barjols, 
Draguignan, du Cannet-des-Maures et de Correns), d'étangs salés (Tourves) ou même de terres salées (Correns).

\section{Commune du Cannet-des-Maures}

5 La commune du Cannet-des-Maures a été choisie pour effectuer les prospections thématiques. La source salée jaillit au lieu-dit Viouret, dans la plaine des Maures à $4 \mathrm{~km}$ au sud-est de la ville. Elle a été aménagée par un puits à une date inconnue. L'utilisation de la source salée pour abreuver les troupeaux est mentionnée au XVIII ${ }^{e}$ s. par un document d'archive. Un certain nombre de sites archéologiques sont connus sur la commune du Cannet-des-Maures, mais aucune découverte n'avait été réalisée dans la zone de la source. Il s'agissait donc à la fois de compléter la carte archéologique de la commune et de déterminer d'éventuels regroupements de sites autour de la source salée.

6 Les prospections menées de septembre à novembre 2007 ont permis de découvrir quatorze sites ou indices de sites archéologiques dans les champs de vigne dans un rayon de $200 \mathrm{~m}$ à $2 \mathrm{~km}$ autour de la source salée, aux lieux-dits Riautord, Bastide Neuve et Font Salade. Nous exposons les résultats ci-après en distinguant chaque phase chronologique.

\section{Préhistoire}

7 Le Paléolithique moyen est représenté par un racloir au lieu-dit Riautord.

8 Trois sites d'habitat vraisemblablement néolithiques ont été découverts aux lieux-dits Font Salade, Riautord et Bastide Neuve. La datation de ces sites repose essentiellement sur l'association de céramique non tournée et de nombreux silex taillés dont certaines pièces sont datables du Néolithique moyen (à Riautord) (détermination des pièces lithiques effectuée par S. Renault et M. Bailly, ESEP).

9 Pour ces trois sites, le très mauvais état de la céramique ainsi que l'absence de forme caractéristique ne permettent aucune détermination. Un indice de site de la même période est matérialisé par deux silex isolés (lieu-dit Font Salade).

\section{Protohistoire}

10 Un fragment de grès de forme atypique a été ramassé à Riautord. Une cupule y a été aménagée. Cet objet fait penser à un fragment de moule de bronzier. En effet, les moules en pierre de l'âge du Bronze sont souvent façonnés dans du grès, qui résiste bien aux fortes chaleurs. La cupule pourrait renvoyer au système permettant de maintenir face à face les deux parties du moule. Cependant, en l'absence de tout autre mobilier de l'âge du Bronze ou d'autres témoins du travail de ce métal, cet objet est à prendre avec beaucoup de prudence.

11 Deux indices de sites protohistoriques sont représentés par quelques tessons de céramique non tournée isolés au lieu-dit Bastide Neuve. Une prospection fine aux abords n'a pas permis de cerner de concentration de mobilier. 


\section{Antiquité}

12 Deux indices d'occupation antique sont représentés par des fragments de tegulae isolés aux lieux-dits Bastide Neuve et Font Salade. Trois concentrations plus denses de tegulae et imbrices en association avec quelques tessons de céramique commune à pâte claire pourraient renvoyer à des sites d'habitats antiques aux lieux-dits Font Salade, Bastide Neuve et Riautord. Ce dernier site comprend quelques fragments de tegulae surcuites et pourrait donc évoquer la présence d'un atelier de tuilier en association avec un habitat.

\section{Conclusion}

Ces découvertes permettent donc d'enrichir la carte archéologique de cette zone de la plaine des Maures où l'on avait jusqu'alors recensé peu de sites.

En outre, la densité d'occupation diachronique mise en évidence aux alentours de la source salée du Cannet-des-Maures est encourageante pour envisager son exploitation durant les périodes anciennes, qu'il s'agira de démontrer en 2008 grâce à des opérations de carottages.

\section{Département du Vaucluse}

Des travaux documentaires avaient permis de montrer la richesse des ressources en sel continental du département, qu'il s'agisse de sources salées (communes de Beaumes-deVenise, Malaucène), d'étang salé (Courthézon) ou même de terres salées (Monteux).

\section{Commune de Beaumes-de-Venise}

La commune de Beaumes-de-Venise a été choisie pour effectuer les prospections thématiques. Quatre sources salées jaillissent dans la vallée de la Salette à $2 \mathrm{~km}$ au nord de la ville. L'une d'entre elles a fait l'objet d'une tentative d'exploitation du sel au XVIII ${ }^{\mathrm{e}} \mathrm{s}$. Un certain nombre de sites archéologiques sont connus sur la commune de Beaumes-deVenise, mais aucune découverte n'avait été réalisée jusqu'alors dans les environs immédiats des sources. Il s'agissait donc à la fois de compléter la carte archéologique de la commune et de déterminer d'éventuels regroupements de sites autour des sources salées.

Les prospections menées de septembre à novembre 2007 ont permis de découvrir neuf sites ou indices de sites archéologiques dans les champs de vigne dans un rayon de $10 \mathrm{~m}$ à $3 \mathrm{~km}$ autour des sources.

\section{Préhistoire}

18 Les prospections aux alentours moins immédiats des quatre sources de Beaumes-deVenise ont révélé quelques indices d'occupation. Un fragment de nucléus et un fragment d'éclat de silex préhistorique ont été découverts sur la montagne de Coyeux, dominant à l'ouest la vallée de la Salette. 


\section{Antiquité tardive} de l'une des sources salées au lieu-dit Le Coulet. Il s'agit d'un mur situé à l'angle d'un chemin et d'un petit vallon, en bas de la colline de la Font Valet. Il est constitué d'un petit appareil irrégulier lié au mortier, incluant aussi une couche de tegulae. Les abords de la structure ont fourni quelques tessons de céramique de l'Antiquité tardive ainsi que des tegulae. La présence de ce site à proximité immédiate d'une des sources salées de Beaumes-de-Venise est donc un facteur plutôt positif pour envisager une exploitation ancienne du sel.

\section{Diachronique}

Les recherches sur le plateau de Saint-Sauveur (lieu-dit Piberat), dominant au sud le débouché de la vallée de la Salette, à environ $3 \mathrm{~km}$ des sources salées, ont montré une occupation allant de la Préhistoire à l'époque moderne. Il s'agit d'éclats de silex préhistoriques, d'un tesson de céramique non tournée protohistorique, de tuiles et de quelques tessons de céramique antiques, et enfin de tuiles modernes dont un raté de cuisson. Une parcelle en contrebas du plateau a également fourni deux indices de sites préhistorique et antique. Enfin, au même endroit, des traces d'araire d'époque indéterminée sont visibles sur un affleurement rocheux.

\section{Conclusion}

Ces découvertes permettent donc d'enrichir la carte archéologique de Beaumes-deVenise, et d'envisager une relation entre certains sites archéologiques et les sources salées de la commune. Des résultats encourageants pour envisager une exploitation du sel durant les périodes anciennes, qu'il s'agira de démontrer en 2008 grâce à des opérations de carottages.

\section{INDEX}

operation Prospection thématique (PRT)

Index chronologique : âge du Bronze, Antiquité tardive, Préhistoire, Protohistoire, Temps

\section{Modernes}

Index géographique : Provence-Alpes-Côte d'Azur, Var (83), Vaucluse (84), Cannet-des-Maures, Beaumes-de-Venise 
AUTEURS

AUDREY BOUTET

AUT 